\title{
INCLUSION RELATIONSHIPS AND SOME INTEGRAL-PRESERVING PROPERTIES OF CERTAIN CLASSES OF MEROMORPHIC P-VALENT FUNCTIONS
}

\author{
S.A. Saleh, A.H. El-Qadeem, M.A. Mamon
}

Abstract. We introduce some integral operators defined on the space of pvalent meromorphic functions in the class $\Sigma_{p}$. By using these integral operators, we define several subclasses of p-valent meromorphic functions and investigate various inclusion relationship and integral-preserving properties.

2010 Mathematics Subject Classification: 30C45.

Keywords: analytic functions, p-valent functions, meromorphic functions, starlike and convex functions, close-to-convex functions, quasi-convex functions, Hadamard product, Rafid operator.

\section{INTRODUCTION}

Let $\Sigma_{p}$ denotes the class of functions $f$ given by

$$
f(z)=\frac{1}{z^{p}}+\sum_{k=1}^{\infty} a_{k-p} z^{k-p} \quad(p \in \mathbb{N}=\{1,2,3, \ldots\})
$$

which are analytic and p-valent in the punctured unit disc

$$
\mathbb{U}^{*}=\{z \in \mathbb{C}: 0<|z|<1\} .
$$

A function $f \in \Sigma_{p}$ is said to be in the class $\Sigma S_{p}^{*}(\alpha)$ of meromorphic p-valent starlike functions of order $\alpha$ in $\mathbb{U}^{*}$ if

$$
\operatorname{Re}\left(\frac{z f^{\prime}(z)}{f(z)}\right)<-\alpha, \quad\left(z \in \mathbb{U}^{*} ; 0 \leq \alpha<p\right),
$$

also, a function $f \in \Sigma_{p}$ is said to be in the class $\Sigma C_{p}(\alpha)$ of meromorphic p-valent convex functions of order $\alpha$ in $\mathbb{U}^{*}$ if

$$
\operatorname{Re}\left(1+\frac{z f^{\prime \prime}(z)}{f^{\prime}(z)}\right)<-\alpha, \quad\left(z \in \mathbb{U}^{*} ; 0 \leq \alpha<p\right) .
$$


It is easy to observe from (2) and (3) that

$$
f \in \Sigma C_{p}(\alpha) \Leftrightarrow-\frac{z f^{\prime}}{p} \in \Sigma S_{p}^{*}(\alpha)
$$

A function $f \in \Sigma_{p}$ is said to be in the class $\Sigma K_{p}(\beta, \alpha)$ of meromorphic pvalent close-to-convex functions of order $\beta$ and type $\alpha$ in $\mathbb{U}^{*}$ if there exist a function $g \in \Sigma S_{p}^{*}(\alpha)$ such that

$$
\operatorname{Re}\left(\frac{z f^{\prime}(z)}{g(z)}\right)<-\beta, \quad\left(z \in \mathbb{U}^{*} ; 0 \leq \alpha, \beta<p\right),
$$

furthermore, a function $f \in \Sigma_{p}$ is said to be in the class $\Sigma K_{p}^{*}(\beta, \alpha)$ of meromorphic p-valent quasi-convex functions of order $\beta$ and type $\alpha$ in $\mathbb{U}^{*}$ if there exist a function $g \in \Sigma C_{p}(\alpha)$ such that

$$
\operatorname{Re}\left(\frac{\left(z f^{\prime}(z)\right)^{\prime}}{g^{\prime}(z)}\right)<-\beta, \quad\left(z \in \mathbb{U}^{*} ; 0 \leq \alpha, \beta<p\right) .
$$

It is easy to observe from (5) and (6) that

$$
f \in \Sigma K_{p}^{*}(\beta, \alpha) \Leftrightarrow-\frac{z f^{\prime}}{p} \in \Sigma K_{p}(\beta, \alpha) .
$$

Definition 1. Let $0 \leq \mu \leq 1 ; 0 \leq \gamma \leq 1 ; p \in \mathbb{N}$ and $f \in \Sigma_{p}$, we introduce the p-valent Rafid operator $S_{\mu, p}^{\gamma}: \Sigma_{p} \rightarrow \Sigma_{p}$ which is defined by

$$
S_{\mu, p}^{\gamma} f(z)=\frac{1}{(1-\mu)^{\gamma+1} \Gamma(\gamma+1)} \int_{0}^{\infty} t^{\gamma+p} e^{\left(-\frac{t}{1-\mu}\right)} f(z t) d t
$$

then,

$$
S_{\mu, p}^{\gamma} f(z)=\frac{1}{z^{p}}+\sum_{k=1}^{\infty} L(\gamma, \mu, k) a_{k-p} z^{k-p}
$$

where,

$$
L(\gamma, \mu, k)=(1-\mu)^{k}(\gamma+1)_{k}
$$

and $(\nu)_{k}$ denotes the Pochhammer symbol given by

$$
(\nu)_{k}=\frac{\Gamma(\nu+k)}{\Gamma(\nu)}= \begin{cases}1 & \text { if } k=0 \\ \nu(\nu+1) \ldots(\nu+k-1) & \text { if } k \in \mathbb{N}\end{cases}
$$


S.A. Saleh, A.H. El-Qadeem, M.A. Mamon - Inclusion Relationships ...

Remark 1. Putting $p=1$ in (8) we have the Rafid operator $S_{\mu}^{\gamma}$ which is introduced by Rosy and Varma [4].

Remark 2. Using the equation (9), it is easy to see that

$$
S_{\mu, p}^{\gamma}\left(z f^{\prime}(z)\right)=z\left(S_{\mu, p}^{\gamma} f(z)\right)^{\prime}
$$

and,

$$
z\left(S_{\mu, p}^{\gamma} f(z)\right)^{\prime}=(\gamma+1) S_{\mu, p}^{\gamma+1} f(z)-(\gamma+p+1) S_{\mu, p}^{\gamma} f(z)
$$

By putting $a_{k-p}=1, \forall k$ in $(9)$, we get

$$
\psi_{\mu, p}^{\gamma}(z)=\frac{1}{z^{p}}+\sum_{k=1}^{\infty} L(\gamma, \mu, k) z^{k-p}
$$

and $\varphi_{\mu, p}^{\gamma, \lambda}(z)$ be defined using the Hadmard product as

$$
\varphi_{\mu, p}^{\gamma, \lambda}(z) * \psi_{\mu, p}^{\gamma}(z)=\frac{1}{z^{p}(1-z)^{\lambda}}
$$

therefore,

$$
\varphi_{\mu, p}^{\gamma, \lambda}(z)=\frac{1}{z^{p}}+\sum_{k=1}^{\infty} \frac{(\lambda)_{k}}{(1-\mu)^{k}(\gamma+1)_{k}} z^{k-p}
$$

Definition 2. For $0 \leq \mu \leq 1,0 \leq \gamma \leq 1, \lambda>0$ and $p \in \mathbb{N}$, we introduce the integral operator $J_{\mu, p}^{\gamma, \lambda}: \Sigma_{p} \rightarrow \Sigma_{p}$ which is defined by

$$
J_{\mu, p}^{\gamma, \lambda} f(z)=\varphi_{\mu, p}^{\gamma, \lambda}(z) * f(z)
$$

Therefore,

$$
J_{\mu, p}^{\gamma, \lambda} f(z)=\frac{1}{z^{p}}+\sum_{k=1}^{\infty} \frac{1}{(1-\mu)^{k}(\gamma+1)_{k}} \frac{(\lambda)_{k}}{(1)_{k}} a_{k-p} z^{k-p}
$$

Remark 3. Using equation (15), it is easy to see that

$$
z\left(J_{\mu, p}^{\gamma+1, \lambda} f(z)\right)^{\prime}=(\gamma+1) J_{\mu, p}^{\gamma, \lambda} f(z)-(p+\gamma+1) J_{\mu, p}^{\gamma+1, \lambda} f(z),
$$

and

$$
z\left(J_{\mu, p}^{\gamma, \lambda} f(z)\right)^{\prime}=\lambda J_{\mu, p}^{\gamma, \lambda+1} f(z)-(p+\lambda) J_{\mu, p}^{\gamma, \lambda} f(z) .
$$


S.A. Saleh, A.H. El-Qadeem, M.A. Mamon - Inclusion Relationships ...

We now define the following subclasses of the meromorphic function class $\Sigma_{p}$ by means of the integral operator $J_{\mu, p}^{\gamma, \lambda}$ given by (14).

$$
\begin{aligned}
\Sigma S_{\mu, p}^{* \gamma, \lambda}(\alpha) & =\left\{f: f \in \Sigma_{p} \text { and } J_{\mu, p}^{\gamma, \lambda} f(z) \in \Sigma S_{p}^{*}(\alpha)\right\} \\
\Sigma C_{\mu, p}^{\gamma, \lambda}(\alpha) & =\left\{f: f \in \Sigma_{p} \text { and } J_{\mu, p}^{\gamma, \lambda} f(z) \in \Sigma C_{p}(\alpha)\right\} \\
\Sigma K_{\mu, p}^{\gamma, \lambda}(\beta, \alpha) & =\left\{f: f \in \Sigma_{p} \text { and } J_{\mu, p}^{\gamma, \lambda} f(z) \in \Sigma K_{p}(\beta, \alpha)\right\} \\
\Sigma K_{\mu, p}^{* \gamma, \lambda}(\beta, \alpha) & =\left\{f: f \in \Sigma_{p} \text { and } J_{\mu, p}^{\gamma, \lambda} f(z) \in \Sigma K_{p}^{*}(\beta, \alpha)\right\}
\end{aligned}
$$

where

$$
z \in \mathbb{U}, 0 \leq \alpha<p, p \in \mathbb{N} .
$$

Before we establish our main result, we need the following lemma due to Miller and Mocanu [3].

Lemma 1. let $\theta(u, v)$ be a complex-valued function such that $\theta: \mathcal{D} \rightarrow \mathbb{C}, \mathcal{D} \subset \mathbb{C} \times \mathbb{C}$ $\left(\mathbb{C}\right.$ is the complex plane) and let $u=u_{1}+i u_{2}$ and $v=v_{1}+i v_{2}$. Suppose that $\theta(u, v)$ satisfies the following conditions:

- $\theta(u, v)$ is continuous in $\mathcal{D}$;

- $(1,0) \in \mathcal{D}$ and $\operatorname{Re}\{\theta(1,0)\}>0$;

- for all $\left(i u_{2}, v_{1}\right) \in \mathcal{D}$ such that $v_{1} \leq \frac{-1}{2}\left(1+u_{2}^{2}\right), \operatorname{Re}\left\{\theta\left(i u_{2}, v_{1}\right)\right\} \leq 0$.

Let,

$$
q(z)=1+q_{1} z+q_{2} z^{2}+\ldots
$$

be an analytic in $U$ such that $\left(q(z), z q^{\prime}(z)\right) \in \mathcal{D}(z \in \mathbb{U})$. If $\operatorname{Re}\left\{\theta\left(q(z), z q^{\prime}(z)\right)\right\}>0$, then $\operatorname{Re}\{q(z)\}>0$.

\section{INCLUSION RELATIONSHIPS}

In this section, we give several inclusion relationships for $\mathrm{p}$-valent meromorphic function classes, which are associated with the integral operator $J_{\mu, p}^{\gamma, \lambda}$.

Theorem 2. Let $0 \leq \mu \leq 1,0 \leq \gamma \leq 1, \lambda>0$ and $0 \leq \alpha<p, p \in \mathbb{N}$, then

$$
\Sigma S_{\mu, p}^{* \gamma, \lambda+1}(\alpha) \subset \Sigma S_{\mu, p}^{* \gamma, \lambda}(\alpha) \subset \Sigma S_{\mu, p}^{* \gamma+1, \lambda}(\alpha)
$$


Proof. (i) We first show that

$$
\Sigma S_{\mu, p}^{* \gamma, \lambda+1}(\alpha) \subset \Sigma S_{\mu, p}^{* \gamma, \lambda}(\alpha)
$$

Let $f(z) \in \Sigma S_{\mu, p}^{* \gamma, \lambda+1}(\alpha)$ and set

$$
\frac{z\left(J_{\mu, p}^{\gamma, \lambda} f(z)\right)^{\prime}}{J_{\mu, p}^{\gamma, \lambda} f(z)}=-\alpha-(p-\alpha) q(z)
$$

where $q(z)$ is given by (22). By using equation (17), we have

$$
\frac{\lambda J_{\mu, p}^{\gamma, \lambda+1} f(z)}{J_{\mu, p}^{\gamma, \lambda} f(z)}=(p+\lambda-\alpha)-(p-\alpha) q(z)
$$

Differentiating (25) logarithmically with respect to $z$, we obtain

$$
\begin{aligned}
\frac{z\left(J_{\mu, p}^{\gamma, \lambda+1} f(z)\right)^{\prime}}{J_{\mu, p}^{\gamma, \lambda+1} f(z)} & =\frac{z\left(J_{\mu, p}^{\gamma, \lambda} f(z)\right)^{\prime}}{J_{\mu, p}^{\gamma, \lambda} f(z)}+\frac{z(p-\alpha) q^{\prime}(z)}{(p-\alpha) q(z)-(p+\lambda-\alpha)} \\
& =-\alpha-(p-\alpha) q(z)+\frac{z(p-\alpha) q^{\prime}(z)}{(p-\alpha) q(z)-(p+\lambda-\alpha)}
\end{aligned}
$$

Let now,

$$
\theta(u, v)=(p-\alpha) u-\frac{(p-\alpha) v}{(p-\alpha) u-(p+\lambda-\alpha)}
$$

where $u=q(z)=u_{1}+i u_{2}$ and $v=z q^{\prime}(z)=v_{1}+i v_{2}$. Then,

- $\theta(u, v)$ is continuous in $\mathcal{D}=\left\{\mathbb{C} \backslash\left(\frac{p+\lambda-\alpha}{p-\alpha}\right)\right\} \times \mathbb{C}$;

- $(1,0) \in \mathcal{D}$ with $\operatorname{Re}\{\theta(1,0)\}=p-\alpha>0$;

- for all $\left(i u_{2}, v_{1}\right) \in \mathcal{D}$ such that $v_{1} \leq \frac{-1}{2}\left(1+u_{2}^{2}\right)$, we have

$$
\begin{aligned}
\operatorname{Re}\left\{\theta\left(i u_{2}, v_{1}\right)\right\} & =\operatorname{Re}\left\{(p-\alpha) i u_{2}-\frac{(p-\alpha) v_{1}}{(p-\alpha) i u_{2}-(p+\lambda-\alpha)}\right\} \\
& =\operatorname{Re}\left\{\frac{-(p-\alpha) v_{1}}{(p-\alpha) i u_{2}-(p+\lambda-\alpha)} * \frac{-(p-\alpha) i u_{2}-(p+\lambda-\alpha)}{-(p-\alpha) i u_{2}-(p+\lambda-\alpha)}\right\} \\
& =\frac{(p+\lambda-\alpha)(p-\alpha) v_{1}}{\left((p-\alpha) u_{2}\right)^{2}+(p+\lambda-\alpha)^{2}} \\
& \leq-\frac{(p+\lambda-\alpha)(p-\alpha)\left(1+u_{2}^{2}\right)}{2\left[\left((p-\alpha) u_{2}\right)^{2}+(p+\lambda-\alpha)^{2}\right]}<0
\end{aligned}
$$


which shows that $\theta(u, v)$ satisfies the hypotheses of Lemma 1 then $\operatorname{Re} q(z)>0$. Consequently, we easily obtain the inclusion relationship (24).

(ii) by using the similar argument in proving relation (24) together with (16) and $\theta(u, v)$ is continuous in $\mathcal{D}=\left\{\mathbb{C} \backslash\left(\frac{p+\gamma+1-\alpha}{p-\alpha}\right)\right\} \times \mathbb{C}$, we can prove the right part of Theorem 1 that is

$$
\Sigma S_{\mu, p}^{* \gamma, \lambda}(\alpha) \subset \Sigma S_{\mu, p}^{* \gamma+1, \lambda}(\alpha)
$$

By combining the inclusion relationships (24) and (28), we complete the proof of Theorem 1.

Theorem 3. Let $0 \leq \mu \leq 1,0 \leq \gamma \leq 1, \lambda>0$ and $0 \leq \alpha<p, p \in \mathbb{N}$, then

$$
\Sigma C_{\mu, p}^{\gamma, \lambda+1}(\alpha) \subset \Sigma C_{\mu, p}^{\gamma, \lambda}(\alpha) \subset \Sigma C_{\mu, p}^{\gamma+1, \lambda}(\alpha)
$$

Proof. Let $f(z) \in \Sigma C_{\mu, p}^{\gamma, \lambda+1}(\alpha)$. Then, from (18), we have

$$
J_{\mu, p}^{\gamma, \lambda+1} f \in \Sigma C_{p}(\alpha)
$$

Furthermore, in view of (4), we find that

$$
-\frac{z}{p}\left(J_{\mu, p}^{\gamma, \lambda+1} f\right)^{\prime} \in \Sigma S_{p}^{*}(\alpha)
$$

that is,

$$
J_{\mu, p}^{\gamma, \lambda+1}\left(-\frac{z}{p} f^{\prime}\right) \in \Sigma S_{p}^{*}(\alpha)
$$

Therefore,

$$
-\frac{z}{p} f^{\prime} \in \Sigma S_{\mu, p}^{* \gamma, \lambda+1}
$$

In view of Theorem 1 , we have

$$
-\frac{z}{p} f^{\prime} \in \Sigma S_{\mu, p}^{* \gamma, \lambda+1} \subset \Sigma S_{\mu, p}^{* \gamma, \lambda}(\alpha)
$$

Then, we get that $f \in \Sigma C_{\mu, p}^{\gamma, \lambda}(\alpha)$ which implies that,

$$
\Sigma C_{\mu, p}^{\gamma, \lambda+1}(\alpha) \subset \Sigma C_{\mu, p}^{\gamma, \lambda}(\alpha)
$$

The right part of Theorem 2 can be proved using the same arguments. The proof is thus completed.

Theorem 4. Let $0 \leq \mu \leq 1,0 \leq \gamma \leq 1, \lambda>0$ and $0 \leq \alpha<p, p \in \mathbb{N}$, then

$$
\Sigma K_{\mu, p}^{\gamma, \lambda+1}(\beta, \alpha) \subset \Sigma K_{\mu, p}^{\gamma, \lambda}(\beta, \alpha) \subset \Sigma K_{\mu, p}^{\gamma+1, \lambda}(\beta, \alpha)
$$


Proof. (i) let us first prove that

$$
\Sigma K_{\mu, p}^{\gamma, \lambda+1}(\beta, \alpha) \subset \Sigma K_{\mu, p}^{\gamma, \lambda}(\beta, \alpha)
$$

Let $f(z) \in \Sigma K_{\mu, p}^{\gamma, \lambda+1}(\beta, \alpha)$. Then there exists a function $\Omega(z) \in \Sigma S_{p}^{*}(\alpha)$ such that

$$
\operatorname{Re}\left(\frac{z\left(J_{\mu, p}^{\gamma, \lambda+1} f(z)\right)^{\prime}}{\Omega(z)}\right)<-\beta \quad\left(z \in U^{*}\right)
$$

We set

$$
\Omega(z)=J_{\mu, p}^{\gamma, \lambda+1} g(z)
$$

So that we have

$$
g(z) \in \Sigma S_{\mu, p}^{* \gamma, \lambda+1}(\alpha) \text { and } \operatorname{Re}\left(\frac{z\left(J_{\mu, p}^{\gamma, \lambda+1} f(z)\right)^{\prime}}{J_{\mu, p}^{\gamma, \lambda+1} g(z)}\right)<-\beta \quad\left(z \in U^{*}\right)
$$

By setting that,

$$
\frac{z\left(J_{\mu, p}^{\gamma, \lambda} f(z)\right)^{\prime}}{J_{\mu, p}^{\gamma, \lambda} g(z)}=-\beta-(p-\beta) q(z)
$$

where $q(z)$ is given by (22). Then, By using the identity (17), we obtain

$$
\begin{aligned}
& \frac{z\left(J_{\mu, p}^{\gamma, \lambda+1} f(z)\right)^{\prime}}{J_{\mu, p}^{\gamma, \lambda+1} g(z)}= \frac{J_{\mu, p}^{\gamma, \lambda+1}\left(z f^{\prime}(z)\right)}{J_{\mu, p}^{\gamma, \lambda+1} g(z)} \\
&= \frac{z\left(J_{\mu, p}^{\gamma, \lambda}\left(z f^{\prime}(z)\right)\right)^{\prime}+(p+\lambda) J_{\mu, p}^{\gamma, \lambda}\left(z f^{\prime}(z)\right)}{z\left(J_{\mu, p}^{\gamma, \lambda} g(z)\right)^{\prime}+(p+\lambda) J_{\mu, p}^{\gamma, \lambda} g(z)} \\
&= \frac{z\left(J_{\mu, p}^{\gamma, \lambda}\left(z f^{\prime}(z)\right)\right)^{\prime}}{J_{\mu, p}^{\gamma, \lambda} g(z)}+(p+\lambda) \frac{J_{\mu, p}^{\gamma, \lambda}\left(z f^{\prime}(z)\right)}{J_{\mu, p}^{\gamma, \lambda} g(z)} \\
& \frac{z\left(J_{\mu, p}^{\gamma, \lambda} g(z)\right)^{\prime}}{J_{\mu, p}^{\gamma, \lambda} g(z)}+(p+\lambda)
\end{aligned}
$$

Since $g(z) \in \Sigma S_{\mu, p}^{* \gamma, \lambda+1}(\alpha)$, by Theorem 1, we can setting

$$
\frac{z\left(J_{\mu, p}^{\gamma, \lambda} g(z)\right)^{\prime}}{J_{\mu, p}^{\gamma, \lambda} g(z)}=-\alpha-(p-\alpha) H(z)
$$


S.A. Saleh, A.H. El-Qadeem, M.A. Mamon - Inclusion Relationships ...

where $H(z)=g_{1}(x, y)+i g_{2}(x, y)$ and $\operatorname{Re}\{H(z)\}=g_{1}(x, y)>0 \quad\left(z \in U^{*}\right)$. Then,

$$
\frac{z\left(J_{\mu, p}^{\gamma, \lambda+1} f(z)\right)^{\prime}}{J_{\mu, p}^{\gamma, \lambda+1} g(z)}=\frac{\frac{z\left(J_{\mu, p}^{\gamma, \lambda}\left(z f^{\prime}(z)\right)\right)^{\prime}}{J_{\mu, p}^{\gamma, \lambda} g(z)}+(p+\lambda)[-\beta-(p-\beta) q(z)]}{-\alpha-(p-\alpha) H(z)+(p+\lambda)}
$$

Thus we have from (32) that

$$
z\left(J_{\mu, p}^{\gamma, \lambda} f(z)\right)^{\prime}=-J_{\mu, p}^{\gamma, \lambda} g(z)[\beta+(p-\beta) q(z)]
$$

Differentiating both sides of (35) with respect to $z$, we obtain

$$
\begin{aligned}
\frac{z\left(J_{\mu, p}^{\gamma, \lambda} z f^{\prime}(z)\right)^{\prime}}{J_{\mu, p}^{\gamma, \lambda} g(z)} & =-[\beta+(p-\beta) q(z)] \frac{z\left(J_{\mu, p}^{\gamma, \lambda} g(z)\right)^{\prime}}{J_{\mu, p}^{\gamma, \lambda} g(z)}-(p-\beta) z q^{\prime}(z) \\
& =-(p-\beta) z q^{\prime}(z)+[\beta+(p-\beta) q(z)][\alpha+(p-\alpha) H(z)]
\end{aligned}
$$

Now, substituting from (36) into (34), we have

$$
\frac{z\left(J_{\mu, p}^{\gamma, \lambda+1} f(z)\right)^{\prime}}{J_{\mu, p}^{\gamma, \lambda+1} g(z)}=-\beta-(p-\beta) q(z)+\frac{(p-\beta) z q^{\prime}(z)}{(p-\alpha) H(z)+\alpha-(p+\lambda)}
$$

Taking $u=q(z)=u_{1}+i u_{2}$ and $v=z q^{\prime}(z)=v_{1}+i v_{2}$, we define the function $\Phi(u, v)$ by

$$
\Phi(u, v)=(p-\beta) u-\frac{(p-\beta) v}{(p-\alpha) H(z)+\alpha-(p+\lambda)}
$$

where $(u, v) \in \mathcal{D}=\left\{\left(\mathbb{C} \backslash D^{*}\right) \times \mathbb{C}\right\}$ and

$$
D^{*}=\left\{z: z \in \mathbb{C} \text { and } \operatorname{Re}\{H(z)\}=g_{1}(x, y) \geq 1+\frac{\lambda}{p-\alpha}\right\}
$$

Then, it follows from (37) that,

- $\Phi(u, v)$ is continuous in $\mathcal{D}=\left(\mathbb{C} \backslash D^{*}\right) \times \mathbb{C}$;

- $(1,0) \in \mathcal{D}$ with $\operatorname{Re}\{\Phi(1,0)\}=p-\beta>0$; 
S.A. Saleh, A.H. El-Qadeem, M.A. Mamon - Inclusion Relationships ...

- for all $\left(i u_{2}, v_{1}\right) \in \mathcal{D}$ such that $v_{1} \leq \frac{-1}{2}\left(1+u_{2}^{2}\right)$, we have

$$
\begin{aligned}
\operatorname{Re}\left\{\Phi\left(i u_{2}, v_{1}\right)\right\} & =\operatorname{Re}\left\{(p-\beta) i u_{2}-\frac{(p-\beta) v_{1}}{(p-\alpha) H(z)+\alpha-(p+\lambda)}\right\} \\
& =\operatorname{Re}\left\{\frac{-(p-\beta) v_{1}}{i(p-\alpha) g_{2}(x, y)+\left[(p-\alpha) g_{1}(x, y)+\alpha-(p+\lambda)\right]}\right\} \\
& =\frac{(p-\beta)\left[(p+\lambda)-\alpha-(p-\alpha) g_{1}(x, y)\right] v_{1}}{\left[(p-\alpha) g_{2}(x, y)\right]^{2}+\left[(p-\alpha) g_{1}(x, y)+\alpha-(p+\lambda)\right]^{2}} \\
& \leq-\frac{1}{2} \frac{(p-\beta)\left[(p+\lambda)-\alpha-(p-\alpha) g_{1}(x, y)\right]\left(1+u_{2}^{2}\right)}{\left[(p-\alpha) g_{2}(x, y)\right]^{2}+\left[(p-\alpha) g_{1}(x, y)+\alpha-(p+\lambda)\right]^{2}}<0
\end{aligned}
$$

which proves that $\Phi(u, v)$ satisfies the hypotheses of Lemma 1 , then $\operatorname{Re}(q(z))>0$. Thus, in the light of (32), we easily deduce the inclusion relationship (31).

(ii) By using the similar argument in proving relation (31) together with (16) and

$$
D^{*}=\left\{z: z \in \mathbb{C} \text { and } \operatorname{Re}\{H(z)\}=g_{1}(x, y) \geq \frac{\gamma+1-\alpha}{p-\alpha}\right\}
$$

we can prove the right part of Theorem 3 . that is

$$
\Sigma K_{\mu, p}^{\gamma, \lambda}(\beta, \alpha) \subset \Sigma K_{\mu, p}^{\gamma+1, \lambda}(\beta, \alpha)
$$

By combining the inclusion relationships (31) and (38), we complete the proof of Theorem 3.

Theorem 5. Let $0 \leq \mu \leq 1,0 \leq \gamma \leq 1, \lambda>0$ and $0 \leq \alpha<p, p \in \mathbb{N}$, then

$$
\Sigma K_{\mu, p}^{* \gamma, \lambda+1}(\beta, \alpha) \subset \Sigma K_{\mu, p}^{* \gamma, \lambda}(\beta, \alpha) \subset \Sigma K_{\mu, p}^{* \gamma+1, \lambda}(\beta, \alpha)
$$

Proof. Just as we derived Theorem 2 as a consequence of Theorem 1 by using the equivalence (4), we can also use the equivalence (7) to prove this Theorem as a consequence of Theorem 3 .

\section{A SET OF INTEGRAL-PRESERVING PROPERTIES}

In this section, we present some integral-preserving properties of the meromorphic function classes introduced here. We first recall a familiar integral operator $\mathcal{L}_{c, p}$ which introduced by Bernardi [2] defined by

$$
\mathcal{L}_{c, p} f(z)=\frac{c}{z^{c+p}} \int_{0}^{z} t^{c+p-1} f(t) d t \quad\left(f \in \Sigma_{p} ; c>0 ; p \in \mathbb{N}\right)
$$


S.A. Saleh, A.H. El-Qadeem, M.A. Mamon - Inclusion Relationships ...

which satisfies the following relationship:

$$
z\left(J_{\mu, p}^{\gamma, \lambda} \mathcal{L}_{c, p} f(z)\right)^{\prime}=c J_{\mu, p}^{\gamma, \lambda} f(z)-(p+c) J_{\mu, p}^{\gamma, \lambda} \mathcal{L}_{c, p} f(z)
$$

In order to obtain the integral-preserving properties involving the integral operator $\mathcal{L}_{c, p}$, we also need the following lemma which is popularly known as Jack's lemma [1].

Lemma 6. Let $\omega(z)$ be a non-constant function analytic in $U$ with $\omega(0)=0$. If $|\omega(z)|$ attains its maximum value on the circle $|z|=r<1$ at $z_{0}$, then

$$
z_{0} \omega^{\prime}\left(z_{0}\right)=\zeta \omega\left(z_{0}\right)
$$

where $\zeta$ is a real number and $\zeta \geq 1$.

Unless otherwise mentioned, we assume in the reminder of this section that $c, \lambda>0 ; 0 \leq \mu, \gamma \leq 1 ; \zeta \geq 1$ and $0 \leq \alpha, \beta<p, p \in \mathbb{N}$.

Theorem 7. If $f(z) \in \Sigma S_{\mu, p}^{* \gamma, \lambda}(\alpha)$, Then

$$
\mathcal{L}_{c, p} f(z) \in \Sigma S_{\mu, p}^{* \gamma, \lambda}(\alpha) .
$$

Proof. Suppose that $f(z) \in \Sigma S_{\mu, p}^{* \gamma, \lambda}(\alpha)$ and let

$$
\frac{z\left(J_{\mu, p}^{\gamma, \lambda} \mathcal{L}_{c, p} f(z)\right)^{\prime}}{J_{\mu, p}^{\gamma, \lambda} \mathcal{L}_{c, p} f(z)}=-\frac{p+(p-2 \alpha) \omega(z)}{1-\omega(z)}
$$

where $\omega(0)=0$. Then, by using (41) and (44), we have

$$
\frac{J_{\mu, p}^{\gamma, \lambda} f(z)}{J_{\mu, p}^{\gamma, \lambda} \mathcal{L}_{c, p} f(z)}=\frac{c-(2 p+c-2 \alpha) \omega(z)}{c(1-\omega(z))}
$$

which, upon logarithmic differentiation, we get

$$
\begin{aligned}
\frac{z\left(J_{\mu, p}^{\gamma, \lambda} f(z)\right)^{\prime}}{J_{\mu, p}^{\gamma, \lambda} f(z)} & =\frac{z\left(J_{\mu, p}^{\gamma, \lambda} \mathcal{L}_{c, p} f(z)\right)^{\prime}}{J_{\mu, p}^{\gamma, \lambda} \mathcal{L}_{c, p} f(z)}-\frac{(2 p+c-2 \alpha) z \omega^{\prime}(z)}{c-(2 p+c-2 \alpha) \omega(z)}+\frac{z \omega^{\prime}(z)}{1-\omega(z)} \\
& =-\frac{p+(p-2 \alpha) \omega(z)}{1-\omega(z)}-\frac{(2 p+c-2 \alpha) z \omega^{\prime}(z)}{c-(2 p+c-2 \alpha) \omega(z)}+\frac{z \omega^{\prime}(z)}{1-\omega(z)}
\end{aligned}
$$

so that,

$$
\frac{z\left(J_{\mu, p}^{\gamma, \lambda} f(z)\right)^{\prime}}{J_{\mu, p}^{\gamma, \lambda} f(z)}+\alpha=(\alpha-p) \frac{1+\omega(z)}{1-\omega(z)}-\frac{(2 p+c-2 \alpha) z \omega^{\prime}(z)}{c-(2 p+c-2 \alpha) \omega(z)}+\frac{z \omega^{\prime}(z)}{1-\omega(z)}
$$


Now, assuming that $\max _{|z| \leq\left|z_{0}\right|}|\omega(z)|=\left|\omega\left(z_{0}\right)\right|=1,\left(z \in U^{*}\right)$ and applying Jack's lemma, we obtain

$$
z_{0} \omega^{\prime}\left(z_{0}\right)=\zeta \omega\left(z_{0}\right)
$$

If we set $\omega\left(z_{0}\right)=e^{i \theta},(\cos \theta<0)$ in $(47)$ and observe that

$$
\operatorname{Re}\left\{(\alpha-p) \frac{1+\omega(z)}{1-\omega(z)}\right\}=0
$$

then, we obtain

$$
\begin{aligned}
\operatorname{Re}\left\{\frac{z\left(J_{\mu, p}^{\gamma, \lambda} f(z)\right)^{\prime}}{J_{\mu, p}^{\gamma, \lambda} f(z)}+\alpha\right\} & =\operatorname{Re}\left\{\frac{z_{0} \omega^{\prime}\left(z_{0}\right)}{1-\omega\left(z_{0}\right)}-\frac{(2 p+c-2 \alpha) z_{0} \omega^{\prime}\left(z_{0}\right)}{c-(2 p+c-2 \alpha) \omega\left(z_{0}\right)}\right\} \\
& =\operatorname{Re}\left\{\frac{\zeta e^{i \theta}}{1-e^{i \theta}}-\frac{(2 p+c-2 \alpha) \zeta e^{i \theta}}{c-(2 p+c-2 \alpha) e^{i \theta}}\right\} \\
& =\frac{2 \zeta(c+p-\alpha)(p-\alpha)}{c^{2}+(2 p+c-2 \alpha)^{2}-2 c(2 p+c-2 \alpha) \cos \theta} \\
& >0
\end{aligned}
$$

which obviously contradicts the hypothesis $f(z) \in \Sigma S_{\mu, p}^{* \gamma, \lambda}(\alpha)$. Consequently, we can deduce that $|\omega(z)|<1\left(z \in U^{*}\right)$, which, in view of (44), proves the integralpreserving property asserted by Theorem 5 .

Theorem 8. If $f(z) \in \Sigma C_{\mu, p}^{\gamma, \lambda}(\alpha)$, Then

$$
\mathcal{L}_{c, p} f(z) \in \Sigma C_{\mu, p}^{\gamma, \lambda}(\alpha)
$$

Proof. Suppose that $f(z) \in \Sigma C_{\mu, p}^{\gamma, \lambda}(\alpha)$, then

$$
z f^{\prime}(z) \in \Sigma S_{\mu, p}^{* \gamma, \lambda}(\alpha)
$$

by applying Theorem 5 , we have

$$
\mathcal{L}_{c, p}\left(z f^{\prime}(z)\right) \in \Sigma S_{\mu, p}^{* \gamma, \lambda}(\alpha)
$$

and so,

$$
z\left(\mathcal{L}_{c, p} f(z)\right)^{\prime} \in \Sigma S_{\mu, p}^{* \gamma, \lambda}(\alpha)
$$

which is equivalent to,

$$
\mathcal{L}_{c, p} f(z) \in \Sigma C_{\mu, p}^{\gamma, \lambda}(\alpha)
$$

The proof is completed. 
S.A. Saleh, A.H. El-Qadeem, M.A. Mamon - Inclusion Relationships ...

Theorem 9. If $f(z) \in \Sigma K_{\mu, p}^{\gamma, \lambda}(\beta, \alpha)$, Then

$$
\mathcal{L}_{c, p} f(z) \in \Sigma K_{\mu, p}^{\gamma, \lambda}(\beta, \alpha) .
$$

Proof. Suppose that $f(z) \in \Sigma K_{\mu, p}^{\gamma, \lambda}(\beta, \alpha)$. Then, there exist a function $g(z) \in$ $\Sigma S_{\mu, p}^{* \gamma, \lambda}(\alpha)$ such that

$$
\operatorname{Re}\left\{\frac{z\left(J_{\mu, p}^{\gamma, \lambda} f(z)\right)^{\prime}}{J_{\mu, p}^{\gamma, \lambda} g(z)}\right\}<-\alpha
$$

Let us now setting,

$$
\frac{z\left(J_{\mu, p}^{\gamma, \lambda} \mathcal{L}_{c, p} f(z)\right)^{\prime}}{J_{\mu, p}^{\gamma, \lambda} \mathcal{L}_{c, p} g(z)}+\beta=-(p-\beta) q(z)
$$

where $q(z)$ is given by $(22)$, we find from (41) that

$$
\begin{aligned}
\frac{z\left(J_{\mu, p}^{\gamma, \lambda} f(z)\right)^{\prime}}{J_{\mu, p}^{\gamma, \lambda} g(z)}= & \frac{J_{\mu, p}^{\gamma, \lambda}\left(z f^{\prime}(z)\right)}{J_{\mu, p}^{\gamma, \lambda} g(z)} \\
& =\frac{\frac{z\left(J_{\mu, p}^{\gamma, \lambda} \mathcal{L}_{c, p}\left(z f^{\prime}(z)\right)\right)^{\prime}}{J_{\mu, p}^{\gamma, \lambda} \mathcal{L}_{c, p} g(z)}+(p+c) \frac{J_{\mu, p}^{\gamma, \lambda} \mathcal{L}_{c, p}\left(z f^{\prime}(z)\right)}{J_{\mu, p}^{\gamma, \lambda} \mathcal{L}_{c, p} g(z)}}{\frac{z\left(J_{\mu, p}^{\gamma, \lambda} \mathcal{L}_{c, p} g(z)\right)^{\prime}}{J_{\mu, p}^{\gamma, \lambda} \mathcal{L}_{c, p} g(z)}+(p+c)}
\end{aligned}
$$

Since $g(z) \in \Sigma S_{\mu, p}^{* \gamma, \lambda}(\alpha)$, then according to Theorem 5 we have $\mathcal{L}_{c, p} g(z) \in \Sigma S_{\mu, p}^{* \gamma, \lambda}(\alpha)$. Then, we can set

$$
\frac{z\left(J_{\mu, p}^{\gamma, \lambda} \mathcal{L}_{c, p} g(z)\right)^{\prime}}{J_{\mu, p}^{\gamma, \lambda} \mathcal{L}_{c, p} g(z)}+\alpha=-(p-\alpha) Q(z)
$$

where $\operatorname{Re}\{Q(z)\}>0$. Equation (48) can be written as,

$$
J_{\mu, p}^{\gamma, \lambda} \mathcal{L}_{c, p}\left(z f^{\prime}(z)\right)=(-\beta-(p-\beta) q(z)) J_{\mu, p}^{\gamma, \lambda} \mathcal{L}_{c, p} g(z)
$$

By differntioniating both sides of (48) with respect to $z$, we get

$$
\begin{aligned}
\frac{z\left(J_{\mu, p}^{\gamma, \lambda} \mathcal{L}_{c, p}\left(z f^{\prime}(z)\right)\right)^{\prime}}{\left(J_{\mu, p}^{\gamma, \lambda} \mathcal{L}_{c, p} g(z)\right)} & =-z q^{\prime}(z)(p-\beta)+(-\beta-(p-\beta) q(z)) \frac{z\left(J_{\mu, p}^{\gamma, \lambda} \mathcal{L}_{c, p} g(z)\right)^{\prime}}{\left(J_{\mu, p}^{\gamma, \lambda} \mathcal{L}_{c, p} g(z)\right)} \\
& =-z q^{\prime}(z)(p-\beta)+(\beta+(p-\beta) q(z))(\alpha+(p-\alpha) Q(z))
\end{aligned}
$$


S.A. Saleh, A.H. El-Qadeem, M.A. Mamon - Inclusion Relationships ...

Then, by substituting (48), (50) and (52) into (49), we have

$$
\frac{z\left(J_{\mu, p}^{\gamma, \lambda} f(z)\right)^{\prime}}{J_{\mu, p}^{\gamma, \lambda} g(z)}+\beta=-(p-\beta) q(z)-\frac{z q^{\prime}(z)(p-\beta)}{(p+c-\alpha)-(p-\alpha) Q(z)}
$$

Then, by setting $u=q(z)=u_{1}+i u_{2}$ and $v=z q^{\prime}(z)=v_{1}+i v_{2}$, we can define the function $\Omega(u, v)$ by

$$
\Omega(u, v)=-(p-\beta) u-\frac{(p-\beta) v}{(p+c-\alpha)-(p-\alpha) Q(z)}
$$

where $(u, v) \in \mathcal{D} \subset \mathbb{C} \times \mathbb{C}$. The remainder of our proof of Theorem 7 is similar to that of Theorem 3 , so we choose to omit the analogous details involved.

Theorem 10. If $f(z) \in \Sigma K_{\mu, p}^{*, \gamma, \lambda}(\beta, \alpha)$, Then

$$
\mathcal{L}_{c, p} f(z) \in \Sigma K_{\mu, p}^{*, \gamma, \lambda}(\beta, \alpha) .
$$

Proof. Just as we derived Theorem 6 from Theorem 5. Easily, we can deduce Theorem 8 from Theorem 7 . So we choose to omit the proof.

\section{REFERENCES}

[1] I.S. Jack, Functions starlike and convex of ordera, J. London Math. Soc. 3, 2 (1971), 469-474.

[2] S.D. Bernardi, Convex and starlike univalent functions, T. Am. Math. S. 135, (1969), 429-446.

[3] S.S. Miller, P.T. Mocanu, Second order differential inequalities in the complex plane, J. Math. Anal. Appl. 65, (1978), 289-305.

[4] T. Rosy, S. Varma, On a subclass of meromorphic functions defined by Hilbert space operator, Geometry, 2013, Article ID 671826, 4 pages.

\section{S.A. Saleh}

Department of Mathematics, Faculty of Science, Tanta University, Tanta 31527, Egypt email:Dr.sasaleh@hotmail.com

Alaa H. El-Qadeem

Department of Mathematics, Faculty of Science, 
S.A. Saleh, A.H. El-Qadeem, M.A. Mamon - Inclusion Relationships ...

Zagazig University,

Zagazig 44519, Egypt

email: ahhassan@science.zu.edu.eg

Mohamed A. Mamon

Department of Mathematics, Faculty of Science,

Tanta University,

Tanta 31527, Egypt

email: mohamed.saleem@science.tanta.edu.eg 